\title{
A NOVEL HIGH RESOLUTION DOA ESTIMATION DESIGN ALGORITHM OF CLOSE SOURCES SIGNAL FOR UNDERWATER CONDITIONS
}

\author{
Prashil M Junghare ${ }^{1}$, Shoaib Wajid ${ }^{2}$, Cyril Prasanna Raj P. ${ }^{3}$, Richard Lincoln Paulraj ${ }^{4}$ \\ ${ }^{1}$ Research Scholar, PRIST University, Tamilnadu, India \\ ${ }^{2}$ Student, Department of ECE, The Oxford College of Engineering, Karnataka, India \\ ${ }^{3}$ Professor \& Dean, R\&D, M.S. Engineering College, Karnataka, India \\ ${ }^{4}$ Assistant professor, Department of ECE, The Oxford College of Engineering, Karnataka, India
}

\begin{abstract}
Underwater target finding in ocean environment has gain considerable interest in both military and civilian applications. In this paper the performance of directions finding techniques, subspace and the non-subspace methods are presented. In this paper, the Eigen analysis of high resolution and supreme resolution algorithms, comparisons and the performance, resolution analysis are done. The analysis is based on linear array elements and the calculation of the pseudo spectra function of the valuation algorithms. Traditional MUSIC algorithm decomposes the signal covariance matrix and then make the signals subspace obtained is orthogonal to the noise subspace, which decreases the effect of the noise. But when the signals intervals are very small, traditional improved MUSIC algorithm has been unable to distinguish the signals as the SNR decreases. A new improved algorithm is introduced using Singular value decomposition of the covariance matrix. An antenna of ULA configuration is taken for both the algorithms. Simulation results show that projected method gives better performance than MUSIC algorithm. In this newly Modified MUSIC algorithm, conditions required for under water environment are taken into account such as water density, permittivity of water, pressure, Signal to Noise Ratio, speed of sound wave in water.
\end{abstract}

Keywords: Underwater Communication, Number of Snapshots, Antenna Noise, Uniform Linear Array (ULA) and Distance Between Array Elements.

\section{INTRODUCTION}

In signal processing a set of constant parameters on which received signal depends are continuously monitored. DOA estimation carried out using a single fixed antenna has limited resolution, as the physical size of the operating antenna is inversely proportional to the antenna main lobe beam width. It is not practically feasible to increase the size of a single antenna to obtain sharper beam width. An array of antenna sensors provides better performances in parameter estimation and signal reception. So have to use an array of antennas to improve accuracy and resolution. Signal processing aims to process the signals that are received by the sensor array and then strengthen the useful signals by eliminating the noise signals and interference. Array signal processing (ASP) has vital applications in biomedicine, sonar, astronomy, seismic event prediction, wireless communication system, radar etc.

Various algorithms like ESPRIT, MUSIC, WSF, MVDR, ML techniques and others can be used for the estimation process. The entire spatial spectrum is composed of target, observation and estimation stages. It assumes that the signals are distributed in space is in all the directions. So the spatial spectrum of the signal can be exploited to obtain the Direction of Arrival. ESPRIT and MUSIC are the two widely used spectral estimation techniques which work on the principle of decomposition of Eigen values. These subspace based approaches depend on the covariance matrices of the signals.

ESPRIT can be applied to only array structures with some peculiar geometry. Therefore the MUSIC algorithm is the most classic and accepted parameter estimation technique that can be used for both uniform and non-uniform linear arrays. The conventional MUSIC estimation algorithm works on ULA where the array elements are placed in such a way that they satisfy the Nyquist sampling criteria. The design of non- uniform array is quite tedious and it requires various tools. It can compute the number of signals that are being incident on the sensor array, the strength of these signals and the direction i.e. the angle from which the signal are being incident.

\section{RELATED WORK}

With the development of antenna array, the Direction of Arrival (DOA) estimation technique becomes a vital part of smart antenna. The antenna array, which receives number of signals, collecting data at all its array elements with combination of the spatial information, has the ability to process this data optimally and estimate the DOA of impinging signals with high-resolution signal arrival estimation algorithms. Therefore, the high resolution DOA 
estimation algorithm for coherent sources is an essential part of smart array antenna.

MUSIC is an Eigen decomposition algorithm which exploits the underlying information for DOA estimation by decomposing the variance matrix to get eigenvectors and eigenvalues. But due to mutual coupling between the various antenna poles, and due to model error, MUSIC fails to give proper DOA of signals when the noise level is high. MUSIC algorithm fails to differentiate sources which are very closer. The accuracy of the estimation is highly dependent on the type of signal to be detected.

\section{PROPOSED ALGORITHM}

\subsection{Design Considerations:}

- Data formulation for MUSIC \& Improved MUSIC

- Covariance Matrix

- Eigen decomposition.

Compute the MUSIC spectrum

Estimate DOA.

\subsection{Description of the Proposed Music Algorithm:}

MUSIC which is abbreviated as Multiple Signal classification. It is a high resolution technique based on the Eigen-value decomposition of input covariance matrix. It is a simple, popular high resolution and efficient technique. It provides accurate estimation of the angles of arrival, the strengths of signals and the number of signals.

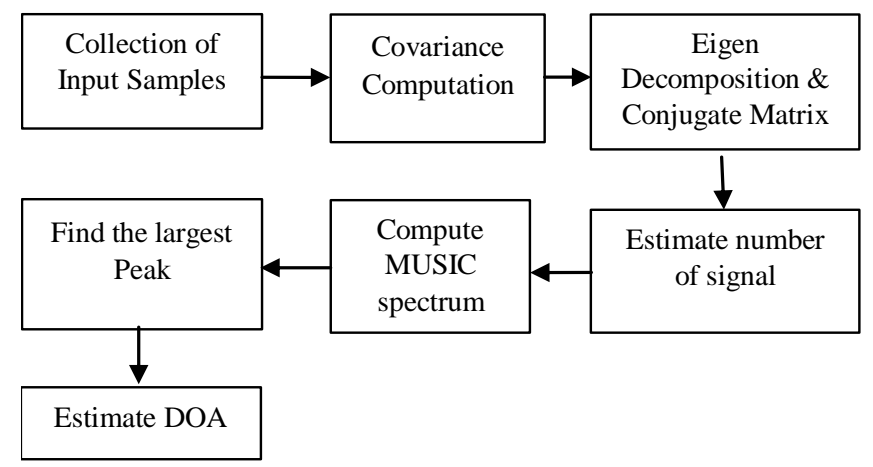

Figure-1: Block Diagram of MUSIC

Figure1, shows the overall calculation of MUSIC algorithm, which has to be followed using the below equations.

Step 1: Take input samples $X_{K}, k=0$ to $N-1 \&$ estimate the input covariance matrix

$$
\hat{R}_{X X}=\frac{1}{k} \sum_{K=0}^{K=-1} X_{K} \cdot X_{K}^{H}
$$

Where $\mathrm{k}$ are number of snapshots, $X_{K}$ is the incoming input signal, $X_{K}^{H}$ is the Signal with Noise.
Step 2: Carryout Eigen decomposition on $\widehat{R}_{x x}$

$$
\hat{R}_{x x} E=E \Lambda
$$

Where $\Lambda=\operatorname{diagof}\left\{\lambda_{0}, \lambda_{1}, \ldots \lambda_{M-1}\right\}$ is Eigen values and Where $\mathrm{E}=\operatorname{diag}\left\{Q_{0}, Q_{1}, \ldots Q_{M-1}\right\}$ is corresponding Eigen vectors of $\hat{R}_{x x}$

Step 3: Calculate the number of signals $\hat{L}$ from multiplicity $\mathrm{K}$, of the smallest eigenvalue $\lambda_{\min }$ in the equation

$$
\widehat{L}=m-k
$$

Step 4: Now we need to compute the MUSIC spectrum by the given equation.

$$
\widehat{P}_{\text {MUSIC }}(\theta)=\frac{A^{H}(\theta) A(\theta)}{A^{H}(\theta) E_{n} E_{n}^{H} A(\theta)}
$$

Step 5: Finally we need to find the $\hat{L}$ largest peaks of $\hat{P}_{\text {MUSIC }}(\theta)$ to obtain estimation of the Direction of Arrival (DOA).

\subsection{Description of the Proposed Improved MUSIC Algorithm:}

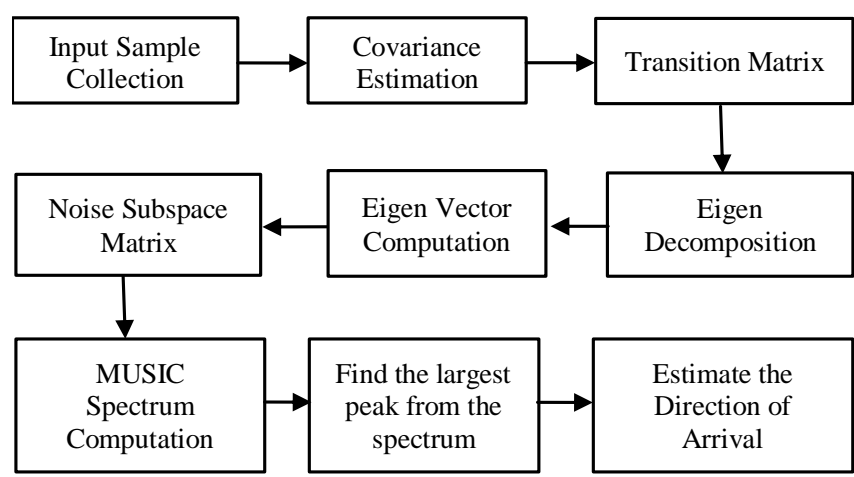

Figure-2: Block Diagram of Improved MUSIC

Figure 2, shows the overall calculation of Improved MUSIC algorithm, which has to be followed using the below equations. MUSIC algorithm has advantages over other estimation algorithm because of the sharp needle spectrum peaks which can efficiently estimate the independent source signals with high precisions unlike the other estimation processes which are limited with low precisions. It has many practical applications as it provides unbiased estimation results. The MUSIC algorithm to estimate the direction has even proved to have better performance in a multiple signal environment. MUSIC algorithm has better resolution, higher precision and accuracy with multiple signals. But this algorithm achieves high resolution in DOA estimation only when the signals being incident on the sensor array are noncoherent. It losses efficiency when the signals are coherent. Keeping all the parameters same as those used for the 
conventional MUSIC in all the previous simulations and considering the coherent signals to be incident on the sensor arrays, obtain the following result.

As the peaks obtain are not sharp and narrow, they fail to estimate the arrival angle for coherent signals. So need to move towards an improved MUSIC algorithm to meet the estimation requirements for coherent signals. To improve results of MUSIC algorithm, we introduce an identity transition matrix ' $\mathrm{T}$ ' and the new received signal matrix $\mathrm{X}$ is given as:

$$
\mathrm{X}=\mathrm{AS}+\mathrm{N}
$$

For an array output $\mathrm{x}$, the corresponding calculations is done to getit's covariance matrix $\mathrm{Rx}$

$$
\text { i.e.. } \mathrm{Rx}=\mathrm{E}\left[\mathrm{XX} \mathrm{H}^{\mathrm{H}}\right]
$$

Where $\mathrm{H}$ is given as the complex conjugate of the original received signal matrix

$$
\begin{gathered}
\mathrm{Rx}=\mathrm{E}\left[(\mathrm{AS}+\mathrm{N}) \cdot(\mathrm{AS}+\mathrm{N})^{\mathrm{H}}\right] \\
\mathrm{AE}\left[\mathrm{SS}^{\mathrm{H}}\right] \mathrm{A}^{\mathrm{H}}+\mathrm{E}\left[\mathrm{NN}^{\mathrm{H}}\right]
\end{gathered}
$$

$\mathrm{ARsA}^{\mathrm{H}}+\mathrm{RN}$

Where $\mathrm{R}_{\mathrm{s}}=\mathrm{E}[\mathrm{SSH}]$,

$\mathrm{RN}=\sigma 2 I$, is Noise correlation matrix.

$\sigma 2$ is Power of noise.

I is unit matrix of $\mathrm{M}^{*} \mathrm{M}$.

Now, consider $\mathrm{Rx}=\mathrm{ARSAH}+\sigma 2 I$,

$\mathrm{Ry}=\mathrm{E}[\mathrm{YYH}]=\mathrm{JRX} * \mathrm{~J}$

Now the matrices Rx and RJ can be summed up to obtain a reconstructed matrix ' $R$ '.As the matrix are summed up they will have the same noise subspaces.

$$
\begin{gathered}
R=R_{x}+R_{y} \\
R=A R_{S} A^{H}+T\left[A R_{S} A^{H}\right] * T+2 \sigma^{2} I
\end{gathered}
$$

According to matrix's equations, the matrices $\mathrm{Rx}, \mathrm{Ry} \& \mathrm{R}$ has got the same noise subspace. Thus conduct characteristics decomposition of R \&obtainits eigenvalue\& eigenvector, according to estimated no. of signal source, we need to separate noise subspaces and then we use this new separated noise subspace to construct spatial spectrum \& get the DOA estimation by finding the peak.

It can be seen that using the improved algorithm for direction of arrival estimation results in narrower peaks for coherent signals. Hence detection of coherent signals can be achieved satisfactorily by the using the improved MUSIC algorithm. MUSIC algorithm fails to obtain narrow and sharp peaks. An Improved version of the MUSIC algorithm as discussed in this paper can be implemented for coherent signals as well. This improved algorithm achieves sharp peaks and makes the estimation process much accurate.

\section{SIMULATION RESULTS}

In this paper, three parameters are considered for output analysis, which includes SNR, number of snapshots and distance between the array elements. Here, three cases are considered, they are:

Case 1: By considering SNR.

Case 2: By considering Number of snapshots.

Case 3: By considering distance b/w the array elements.

By considering all these three cases, here outputs of the MUSIC algorithm and Improved MUSIC are analyzed and their performances are discussed.

\subsection{Simulation of MUSIC:}

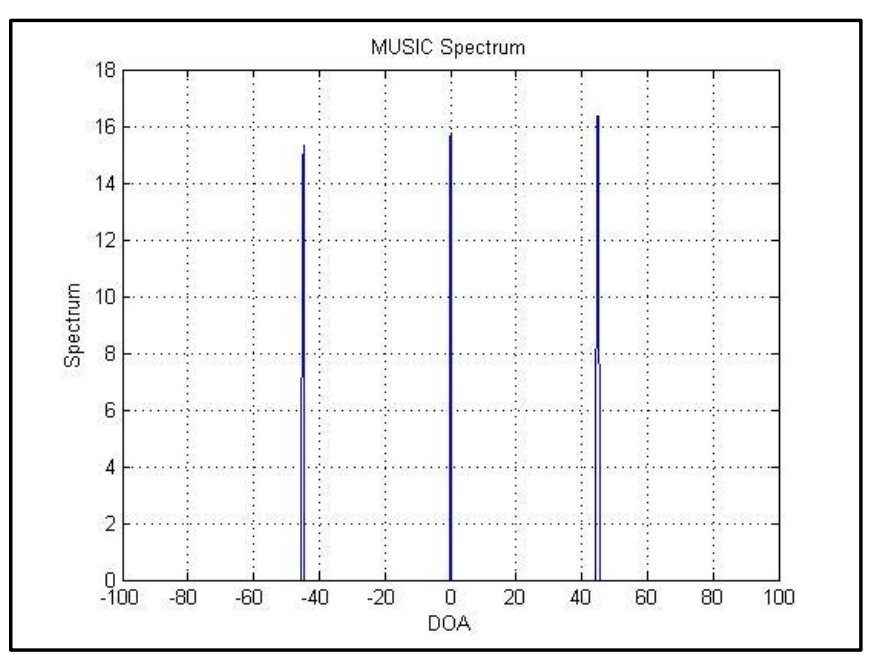

Fig.3: MUSIC Spectrum

The above simulation result shows how MUSIC algorithm identifies the three signals. There are three signals which are independent narrow band signals and the incident angle of these signals are $-45^{\circ}, 0^{\circ}$ and $45^{\circ}$ respectively. These three signals are not correlated, the noise is IGWN(ideal Gaussian white noise) and Signal to noise ratio is assigned as $30 \mathrm{~dB}$, number of array elementsare 12, the no. of Snapshots is given as 100 . Their beam width is very similar. Thus, the no. of array element can be suitably selected according to specific conditions and we make sure of the accuracy of estimation of spectrum. By progressing the speed of operation, work efficiency can be improved.Similar spectrum can be observed for the improved MUSIC with Higher amplitude in figure8.

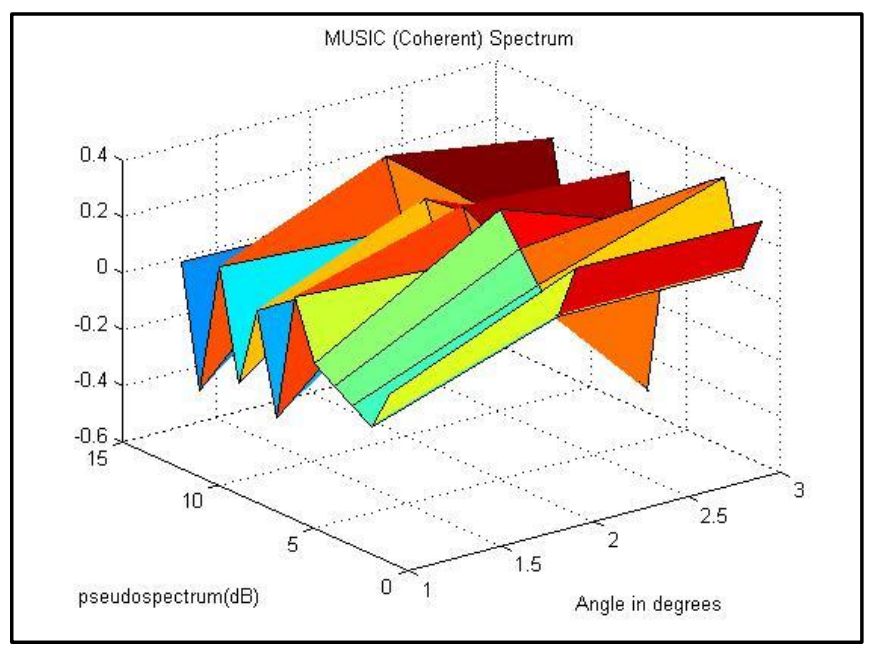

Fig.4: 3D view of coherent spectrum 
As can be seen from Figure4, It is a 3D view of coherent spectrum. Under the above spectrum model shown, the DOA calculation can achieve any accuracy by conquering the conventional inadequacies of low precision; it can take care of direction issues with high resolution \&high precision in numerous sign environment. Thus, high resolution MUSIC calculation may quantify accuracy, higher sensitivity features and potential capability with multi resolution signals, better performance \& higher efficiency, it can give higher resolution and asymptotically balanced DOA calculation, which has animmenseimplication for practical applications.

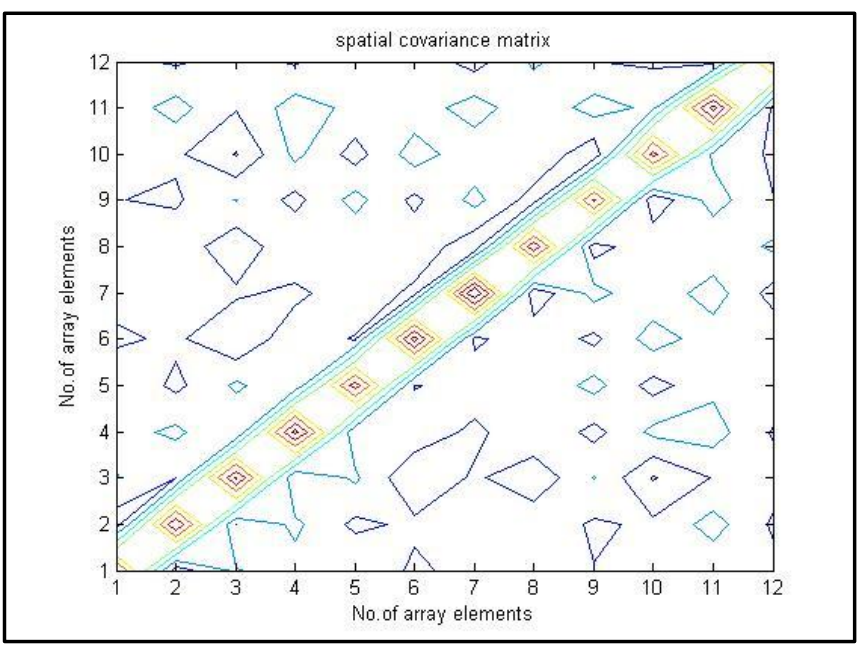

Fig.5: Spatial Covariance Spectrum

The third simulation shows spatial covariance spectrum, As the number of array element increases, there is increase in the spatial covariance, as the spatial covariance increases, there is increase in the noise as well.

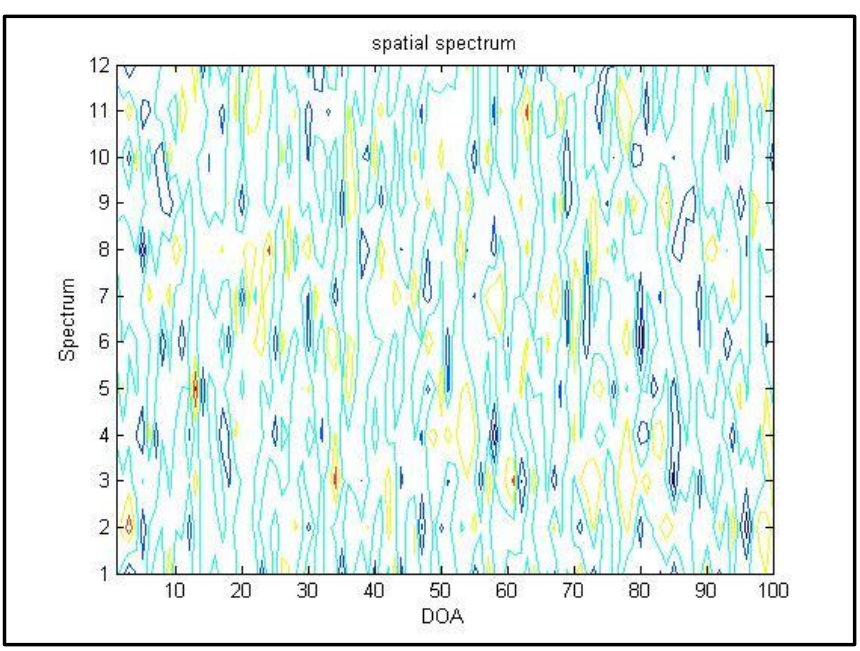

Fig.6: Spatial Spectrum

The above Figure shows a spatial spectrum which is basically an environment noise present while receiving the incoming signal.

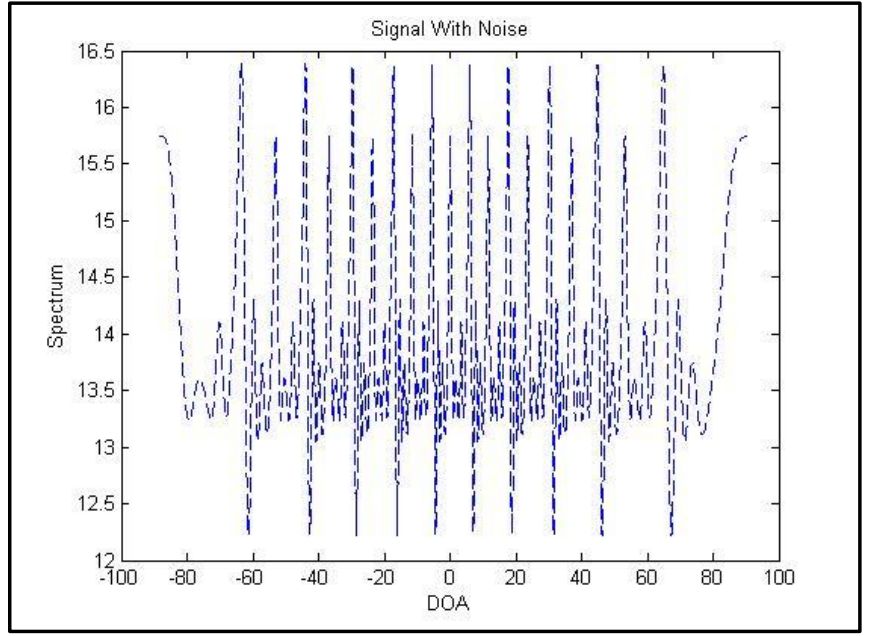

Fig.7: Signal with noise

The fifth simulation figure shows signal with noise, as the name itself tells it's the received signal with the noise present. the incident angle is $-45^{\circ}, 0^{\circ}$ and $45^{\circ}$ respectively.

The signals which are shown beyond these angles are so called noise.

\subsection{Simulation of Improved MUSIC:}

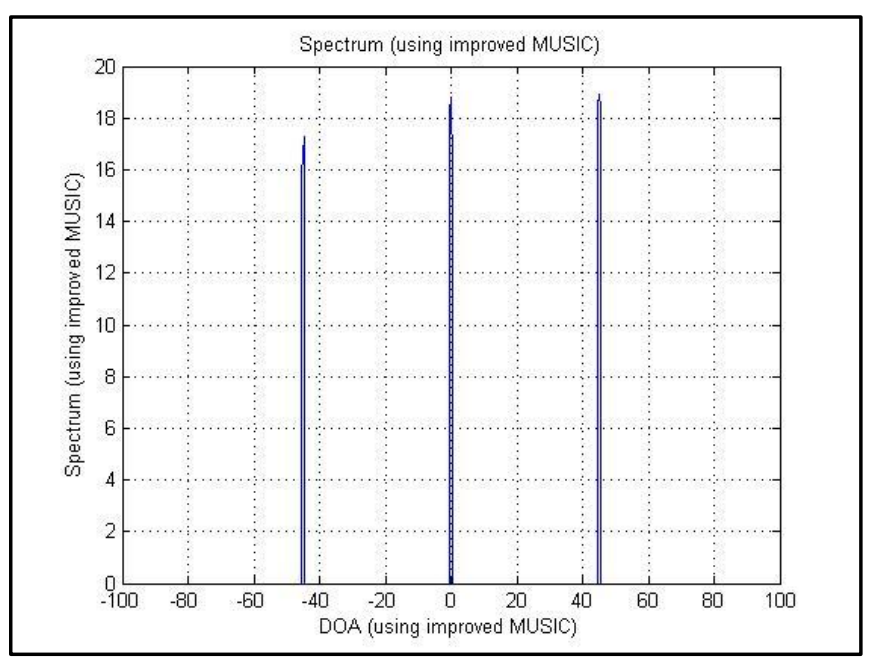

Fig. 8: Improved MUSIC Spectrum

As can be seen from Figure3, this simulation is an improved version of that,There are threeautonomous narrow band signals, for which the incident angle is $-45^{\circ}, 0^{\circ} \& 45^{\circ}$ respectively. The SNR is $30 \mathrm{~dB}$, the number of array element is 12 and the no. of snapshots is 100 . Here in improved MUSIC the amplitude of each signal is increased rapidly. Thus giving high resolution. The simulation results are shown in Figure 8. 


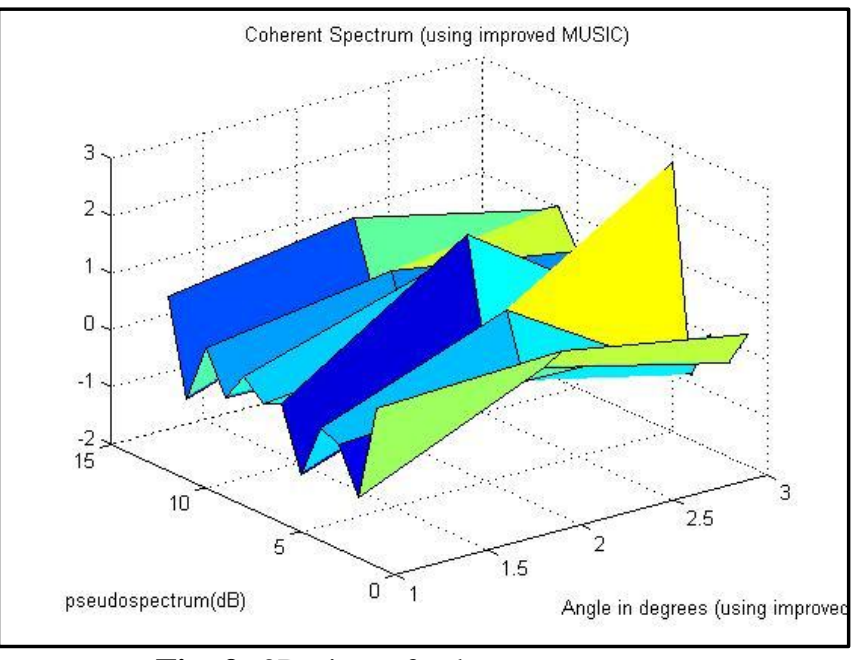

Fig. 9: 3D view of coherent spectrum

As can be seen from Figure4, It is an improved 3D view of coherent spectrum. Where the pseudo spectrum value increases as increase in the amplitude. Thus new improved Music can be better connected to remove the signal correlation trait, which can recognize the coherent signals and calculate the angle of arrival more precisely. Using improvedMusic algorithm, DOA can get high resolution while Music algorithm only concentrate on uncorrelated signals.

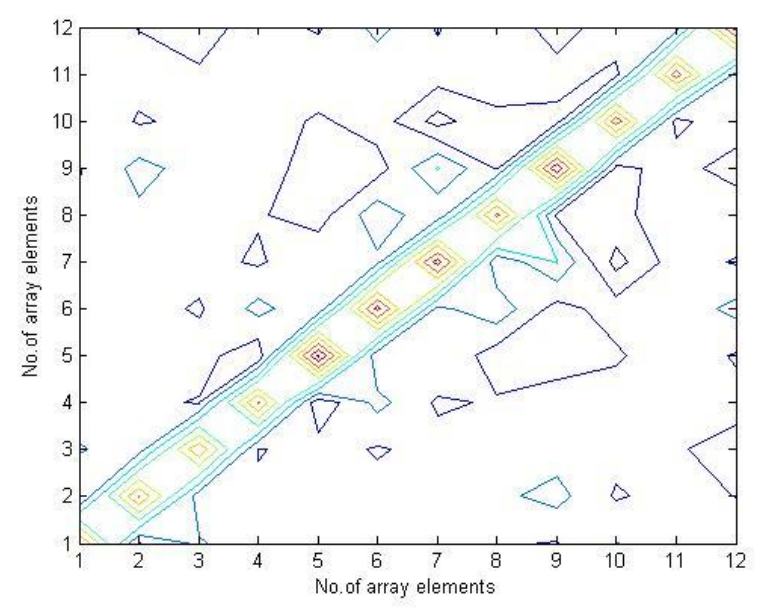

Fig.10: Spatial Covariance Spectrum

The above simulation shows spatial covariance spectrum, where the noises are reduced due to increase in the amplitude. The new Improved MUSIC algorithm can provide DOA calculation more accurately \&will have an effect both on theoretical and practical applications.

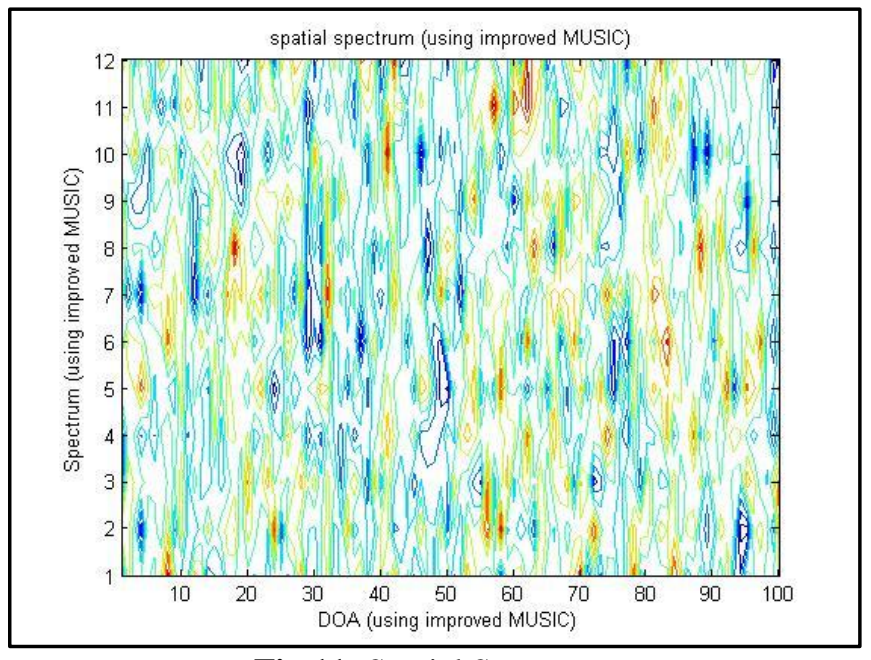

Fig.11: Spatial Spectrum

As can be seen from Figure6, the above Figure shows a spatial spectrum of Improved MUSIC which is basically an environment noise present while receiving the incoming signal.

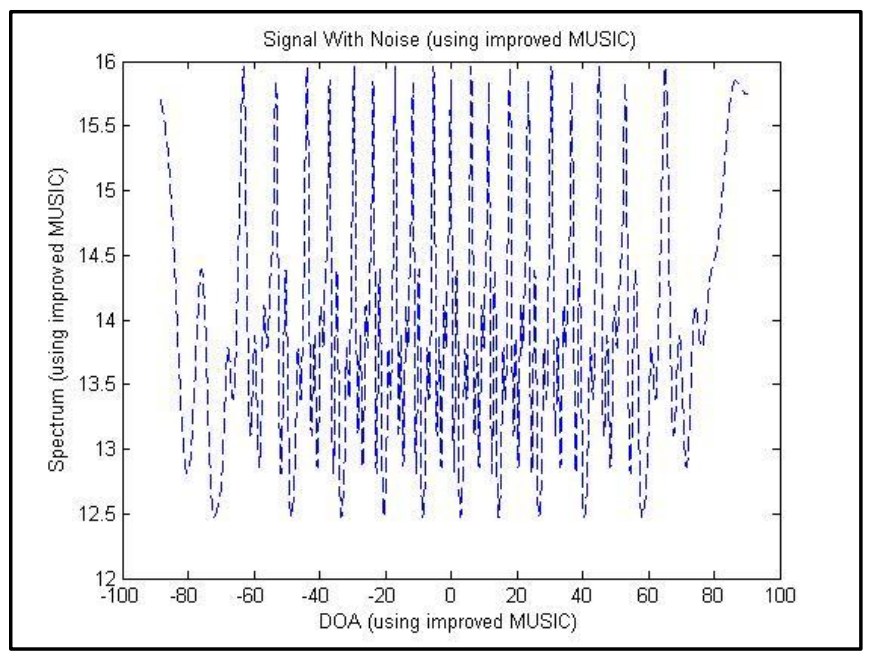

Fig.12: Signal with noise

As can be seen from Figure7, Fig.12 is improved music signal with noise. The dashed line shows the noises and the other remaining conditions unchanged. While in the increase in the no. of noises, the beam width of DOA calculation becomes narrower and thusthe direction of signals becomes clearer and sharper, the accuracy of Music algorithm is also increased. The value of SNR can affect the performance of high resolution DOA calculation. The simulation results are shown in figure12.

\section{CONCLUSION}

I have researched the algorithms of Direction of arrival estimation, found out its limitations and also the effect of array geometries. We have also developed a new algorithm to overcome some challenges provided by the previous algorithm. Based on this research we came to the following conclusions. A widely used MUSIC algorithm was developed and its performance was analyzed in terms of accuracy and resolution. The ULA antenna geometry pattern 
used commonly for estimating DOAs have been studied and its merits and disadvantages were considered compared to UCA. ULA gives an edge over UCA in terms of its simplicity but it only gives azimuth angle, while UCA can give both azimuth and elevation angle. The proposed algorithm for estimating DOA was developed after carrying out certain modifications in MUSIC algorithm, by processing the covariance matrix of the array output signal.

MUSIC algorithm works fine for low level noise regions, but when the noise level is increased its performance starts to degrade. MUSIC also fails to detect signals which are very close by. The new improved algorithm works fine even when the noise level is increased to a certain level. In such levels the algorithm starts to detect noise as desired signals. Thus we get more peaks in signal spectrum graph. In terms of computational complexity, MUSIC tends to be easier as compared to our proposed algorithm. Thus, to get better DOA resolution the scanning rate by the search vectors should be small, which certainly results in a high computational complexity

The application of both the proposed algorithm and MUSIC is limited to the signal sources which are less than the no. of array elements. These techniques uses subspace calculation and Eigen decomposition methods to which it leads to higer complexity. Therefore it limits the use of applications where fast DOA calculation is not necessary. In these both Music and improved music algorithm that I have analyzed can only give azimuth angles, but not the elevation angle, since we have used ULA \& not UCA.

\section{REFERENCES}

[1].Haykin S, Reilly J P, Vertaschitsch E. Some Aspects of Array Signal Processing.IEEE Proc. F, 1992, 139; p1 26.

[2].Haykin S. Array Signal Processing. Prentice Hall. 1985.

[3].S.Unnikersnna Pillai. Array Signal Processing, Springer verlag, 1989.

[4].HongY Wang, Modern Spectrum Estimation, Dongnan University Press, 1990.

[5].Xianci Xiao, Modern Spatial Spectrum Estimation, Haierbin Industry University Press, 1992.

[6].Xianda Zhang, Zheng Bao, Communication Signal Processing, National Defence Industry Press, 2000.

[7].Xianda Zhang, Modern Signal Processing, Tsinghua Press, 2000.

[8].PerteStoica, Maximum Likelihood Method for Direction of Arrival Estimation. IEEE Trans on ASSP. 1990. Vol. 38(7). P1132 1143.

[9]. Michael L. Miller. Maximum Likelihood Narrow-band Direction Finding and EM Algorithms.IEEE Trans on ASSP. 1990. Vol. 36(10). P1560 1577.

[10].Ziskind I. MAX M, Maximum Likelihood Localization of Multiple Sources by Alternating Projection. IEEE Trans on ASSP. 1988. Vol. 36(10). P1553 1560.

[11]. Ronald D, Degrot. The Constrained MUSIC Problem.IEEE Trans on SP.1993.Vol. 41(3).P1445 1449.

[12].Fuli Richard. Analysis of Min-norm and MUSIC with Arbitrary Array Geometry.IEEE Trans on AES.1990.Vol. 26(6).P976 985.

[13] Richard Roy, ThonasKailath. ESPRIT-Estimation of Signal Parameters via Rotational Invariance
Techniques.IEEE Trans on Acoustics Speech and Signal Processing. July 1989. Vol. 37.No.7 pp 984 995.

[14] L.N Yang. Study of Factors Affecting Accuracy of DOA.Modern Rader. June 2007. Vol. 29.No. 6.pp 70-3. [15] C.H Yu, J.L Li. A White Noise Filtering Method for DOA Estimation of Coherent Signals in Low SNR.Signal Processing. July 2012. Vol. 28.No. 7.pp 957-62.

[16] Gilat Amos. MATLAB: an Introduction with Application. 3rd edition.Wiley; John Wiley.Cop 2008.

[17] Chapman, Stephen J. Matlab Programming for Engineers. Pacific Grove, Calif, 2000. Chapter 1, section 1. P 2-3.

[18] Hill, David R. Modern Matrix Algebra. Upper Saddle River, NJ, London: PrenticeHall International, 2001.

[19] Ben Danforth. Variance-Covariance Matrix. June 1, 2009.

[20] Ralph O, Schmidt. Multiple Emitter Location and signal Parameter Estimation. IEEE Trans. On Antennas and Propagation, March 1986. Vol. 34.No. 3. pp 276-280.

[21] DebasisKundu. Modified MUSIC Algorithm for estimating DOA of signals.Department of Mathematics Indian Institute of Technology, kanour, India. November 1993.

[22] Fei Wen, Qun Wan, Rong Fan, Hewen Wei. Improved MUSIC Algorithm for Multiple NoncoherentSubarrays.IEEE Signal Processing Letters. Vol. 21, no. 5, May, 2014. [23] Chen, Xiaoming. "Using Akaike information criterion for selecting the field distribution in a reverberation chamber." Electromagnetic Compatibility, IEEE Transactions on 55.4 (2013): 664-670.

[24] Eder, Rafael, and Johannes Gerstmayr. "Special Genetic Identification Algorithm with smoothing in the frequency domain." Advances in Engineering Software 70 (2014): 113-122.

\section{BIOGRAPHIES}

${ }^{1}$ Mr. Prashil M. Junghare has completed his postgraduation degree (M.Tech) in communication engineering from VIT University in 2009. He currently pursuing Ph.D. Degree from PRIST University, Tamilnadu. His area of interest in underwater sensor and navigation.

\begin{abstract}
${ }^{2}$ Mr.Shoaib Wajid, Student, Dept. of ECE, pursuingM.Tech in VLSI Design \& Embedded Systems, The Oxford College of Engineering, Bangalore, India. His area of interest in the field of Communication, Signal Processing, VLSI. He has done his internship in Karnataka Power Corporation Limited (KPCL) and has attended various Conferences.
\end{abstract}

${ }^{3}$ Dr.Cyril Prasanna Raj P, Currently working as a Dean (R\&D) at M.S Engineering College, Bangalore. He has completed his Ph.D. degree from Coventry University, UK in 2011. His area of interest in VLSI, Image \& signal processing.

${ }^{4}$ Mr. Richard Lincoln Paulraj, Currently working as Assistant professor, Dept. of ECE, The Oxford College of Engineering, Bangalore, India. $\mathrm{He}$ has completed his M.Tech degree from JnanaSangama, Visvesvaraya Technological University, Belagavi, India, in 2011. His area of interest in Networking, Communication, VLSI. 